\title{
Pharmacokinetic/Pharmacodynamic Analysis the Probability of Target Attainment of Posaconazole against Mucorales Species in Patients with Mucormycosis
}

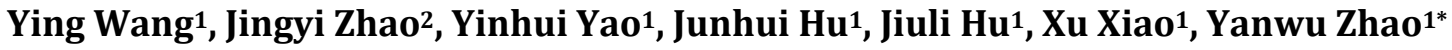 \\ ${ }^{1}$ Department of Pharmacy, the Affiliated Hospital of Chengde Medical College, Chengde, China \\ ${ }^{2}$ Department of Functional Center, Chengde Medical College, Chengde, China \\ Email: *cyfyzyw@163.com, zhaoyw76@126.com
}

How to cite this paper: Wang, Y., Zhao, J.Y., Yao, Y.H., Hu, J.H., Hu, J.L., Xiao, X. and Zhao, Y.W. (2020) Pharmacokinetic/Pharmacodynamic Analysis the Probability of Target Attainment of Posaconazole against Mucorales Species in Patients with Mucormycosis. Pharmacology \& Pharmacy, $11,71-78$

https://doi.org/10.4236/pp.2020.115007

Received: April 18, 2020

Accepted: May 12, 2020

Published: May 15, 2020

Copyright $\odot 2020$ by author(s) and Scientific Research Publishing Inc. This work is licensed under the Creative Commons Attribution International License (CC BY 4.0).

http://creativecommons.org/licenses/by/4.0/

\begin{abstract}
The objective of this study was to investigate the probability of target attainment of various posaconazole dosing regimens against Mucorales species in patients with mucormycosis. According to pharmacokinetic/pharmacodynamic parameters of posaconazole in adults, the dosage regimen of posaconazole for mucormycosis included 50, 100, 200 and $400 \mathrm{mg}$ orally q12h. Monte Carlo Simulation analysed the published parameters of pharmacokinetics and the MIC values of mucormycosis in Mucorales species. The results showed that posaconazole did not affect Rhizopus arrhizus and Mucor sp. The optimal dosage of posaconazole for Rhizopus microsporus and Rhizomucor pusillus was $400 \mathrm{mg}$ orally $\mathrm{q} 12 \mathrm{~h}$ and the best dosage regimen for Lichtheimia corymbifera was $200 \mathrm{mg}$ orally q12h. The antifungal activity of posaconazole against mucormycosis was different, and the dosage regimen needs to adjust according to fungal species.
\end{abstract}

\section{Keywords}

Mote Carlo Simulation, Posaconazole, Mucorales, Mucormycosis

\section{Introduction}

Mucormycosis was a fatal fungal infection caused by Mucorales, which was a subdivision of mucoromycotina. Mucormycosis was the second common fungal infection in hematological malignancies and organ transplantation [1]. It has been reported more and more not only in patients with diabetes or ketoacidosis but also after trauma in patients with normal immune function, even in healthy 
people with food-borne outbreaks caused by Trichoderma [2] [3] [4] [5]. There were many kinds of pathogens creating mucormycosis, such as Rhizopus, Mucor, Lichtheimia, Apophysomyces and Saksenaea, and so on [3] [6].

Posaconazole was the first triazole drug to treat mucormycosis, which was used in refractory mucormycosis or polyene resistant patients [7]. The pharmacological mechanism of posaconazole on fungal infection was that the affinity of fungal P450 demethylase (CYP51) increases, which leads to the rise of penetration or decrease of outflow of the fungal cell membrane [8]. Currently, there are limited reports on the treatment of mucormycosis by the dose of posaconazole, with the maximum daily dose of $800 \mathrm{mg}$ [9]. Therefore, in this study, the administration scheme of posaconazole for the treatment of mucormycosis needs to be optimized, and the optimal drug administration scheme optimised to provide a basis for clinical application.

\section{Materials and Methods}

\subsection{Pharmacokinetic Parameter}

The pharmacokinetic (PK) parameters of posaconazole were derived from the previously published literature [10]. The research reported that the age of people was from 18 to 44 years old, and four different dosages of posaconazole included $50,100,200$ and $400 \mathrm{mg}$ orally q12h. The PK data of posaconazole was established according to the blood drug concentration at different time in the previously published literature, and those data were summarised in Table 1.

\subsection{Minimum Inhibition Concentration (MIC) Distribution of Mucorales}

The MICs data for Mucorales were obtained from Andrew M. et al. [11]. Posaconazole was assessed against 238 isolates of Mucorales using Clinical and Laboratory Standards Institute (CLSI) broth microdilution methods (see Table 2). The number of MIC distributions of posaconazole against Lichtheimia corymbifera, Rhizopus arrhizus, Rhizopus microsporus, Mucor sp. and Rhizomucor pusillus were gathered and were used for each simulation to calculate the probability of target attainment (PTA) and the cumulative fraction of response (CFR).

\subsection{Monte Carlo Simulation}

Briefly, 10,000-subject Monte Carlo simulations were performed to calculate the PTA of each dosage through the PK/PD parameters of posaconazole and MICs distribution of Mucorales. The CFR also was computed as the weighted average of the PTAs across the MIC strata, as shown below [12]:

$$
\mathrm{CFR}=\sum_{i=1}^{n} \mathrm{PTAi} \times \mathrm{Fi}
$$

PTAi is the probability of estimating the target value at a specific MIC; Fi is the probability of each MIC distribution under a certain fungal sample size. Posaconazole activities against Mucorales are concentration-dependent and correlate with 
Table 1. PK parameters of posaconazole in healthy adults.

\begin{tabular}{ccccc}
\hline \multirow{2}{*}{ Parameters } & \multicolumn{4}{c}{ Posaconazole (orally q12h) } \\
\cline { 2 - 5 } & $50 \mathrm{mg}$ & $100 \mathrm{mg}$ & $200 \mathrm{mg}$ & $400 \mathrm{mg}$ \\
\hline $\mathrm{AUC}_{0-24}(\mathrm{ng} \mathrm{h} / \mathrm{ml})$ & $8295(36)$ & $21,778(40)$ & $31,106(26)$ & $73,105(20)$ \\
$\mathrm{t}_{1 / 2}(\mathrm{~h})$ & $19.2(16)$ & $24.1(20)$ & $23.9(26)$ & $31.0(46)$ \\
$\mathrm{CL}(\mathrm{L} / \mathrm{h})$ & $13.5(34)$ & $10.3(32)$ & $13.9(34)$ & $11.5(25)$ \\
\hline
\end{tabular}

Table 2. MICs distribution of posaconazole for 5 Mucorales.

\begin{tabular}{cccccccccccc}
\hline \multirow{2}{*}{ Species } & $\mathrm{n}$ & \multicolumn{10}{c}{$\mathrm{MIC}(\mu \mathrm{g} / \mathrm{ml})$} \\
\cline { 3 - 12 } & & 0.032 & 0.064 & 0.125 & 0.25 & 0.5 & 1 & 2 & 4 & 8 & 16 \\
\hline Lichtheimia corymbifera & 58 & 0 & 3 & 16 & 26 & 13 & 0 & 0 & 0 & 0 & 0 \\
Rhizopus arrhizus & 20 & 0 & 0 & 2 & 3 & 6 & 7 & 0 & 0 & 0 & 2 \\
Rhizopus microsporus & 49 & 0 & 1 & 1 & 13 & 27 & 1 & 3 & 2 & 0 & 1 \\
Mucor sp. & 79 & 3 & 3 & 3 & 9 & 18 & 21 & 10 & 6 & 1 & 5 \\
Rhizomucor pusillus & 32 & 0 & 7 & 2 & 5 & 12 & 4 & 1 & 1 & 0 & 0 \\
\hline
\end{tabular}

the AUC/MIC. For Mucorales, the free-drug 24-h AUC/MIC (fAUC/MIC) was set more than one as the target value. A CFR value of $\geq 90 \%$ was considered to be the minimum for achieving optimal empirical therapy [9]. The binding rate of serum protein of posaconazole was $98 \%$ [13]. The application software is crystal ball (version 11.1.2.4.600, Oracle), in which the free and dose of posaconazole are uniformly distributed, clearance is normal log distribution, MIC is custom distribution.

\section{Results}

\subsection{Probability of Target Attainment (PTA) Analysis}

In this study, the results Monte Carlo simulation of four different administration dosage of posaconazole showed that the patients with mucormycosis meet the requirement of PTA $\geq 90 \%$ in the dosage of $400 \mathrm{mg}$ orally q12h. However, the dosage of posaconazole $50 \mathrm{mg}$ could not be up to standard. The results of dosage of posaconazole $100 \mathrm{mg}$ showed that PTA $\geq 90 \%$ at the highest MIC values for Lichtheimia corymbifera, Rhizopus arrhizus, Rhizopus microsporus, Mucor sp., and Rhizomucor pusillus were $0.125 \mu \mathrm{g} / \mathrm{mL}, 0.25 \mu \mathrm{g} / \mathrm{mL}, 0.25 \mu \mathrm{g} / \mathrm{mL}, 0.125$ $\mu \mathrm{g} / \mathrm{mL}, 0.25 \mu \mathrm{g} / \mathrm{mL}$, respectively. Also, the highest MIC values of $200 \mathrm{mg}$ posaconazole for five Mucorales (Lichtheimia corymbifera, Rhizopus arrhizus, Rhizopus microsporus, Mucor sp., and Rhizomucor pusillus) were PTA more than $90 \%$ at $0.25 \mu \mathrm{g} / \mathrm{mL}, 0.5 \mu \mathrm{g} / \mathrm{mL}, 0.5 \mu \mathrm{g} / \mathrm{mL}, 0.25 \mu \mathrm{g} / \mathrm{mL}, 0.25 \mu \mathrm{g} / \mathrm{mL}$, respectively (see Figure 1).

\subsection{Cumulative Fraction of Response (CFR) Analysis}

The CFR values of each dose regimen simulated by Monte Carlo were shown in Table 3. Rhizopus arrhizus and Mucor sp. had not the bacterial effect on CFR in 
each dose regimen of posaconazole. When the dosage of posaconazole were administered $400 \mathrm{mg}$ orally $\mathrm{q} 12 \mathrm{~h}$ in Rhizopus microsporus, and Rhizomucor pusillus, the CFR values with more than $90 \%$ indicated that posaconazole had a good effect on the two Mucorales mentioned above. When the dosage of posaconazole were $200 \mathrm{mg}$ bid and $400 \mathrm{mg}$ orally q12h, those CFR were more than $90 \%$.

Table 3. CFR (\%) of posaconazole in patients with mucormycosis.

\begin{tabular}{ccccc}
\hline \multirow{2}{*}{ Species } & \multicolumn{4}{c}{$\begin{array}{c}\text { The different administration dosage of } \\
\text { posaconazole orally q12h (CFR, \%) }\end{array}$} \\
\cline { 2 - 4 } & $50 \mathrm{mg}$ & $100 \mathrm{mg}$ & $200 \mathrm{mg}$ & $400 \mathrm{mg}$ \\
\hline Lichtheimia corymbifera & 34.95 & 66.89 & 95.89 & 100 \\
Rhizopus arrhizus & 9.26 & 28.63 & 55.07 & 88.60 \\
Rhizopus microsporus & 7.34 & 52.80 & 85.32 & 90.03 \\
Mucor sp. & 10.95 & 25.53 & 42.91 & 71.53 \\
Rhizomucor pusillus & 27.19 & 48.23 & 74.21 & 93.37 \\
\hline
\end{tabular}

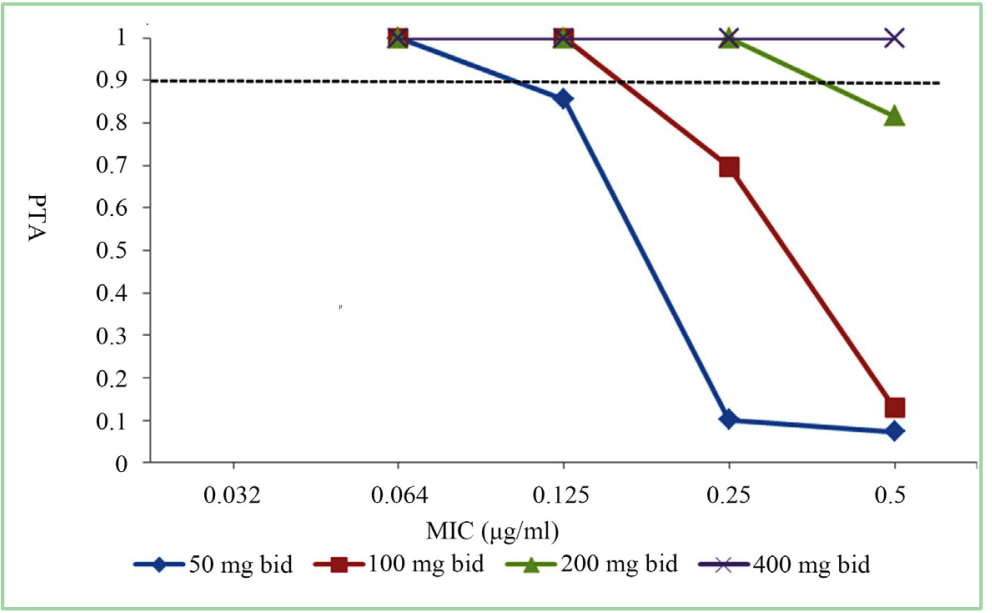

(a)

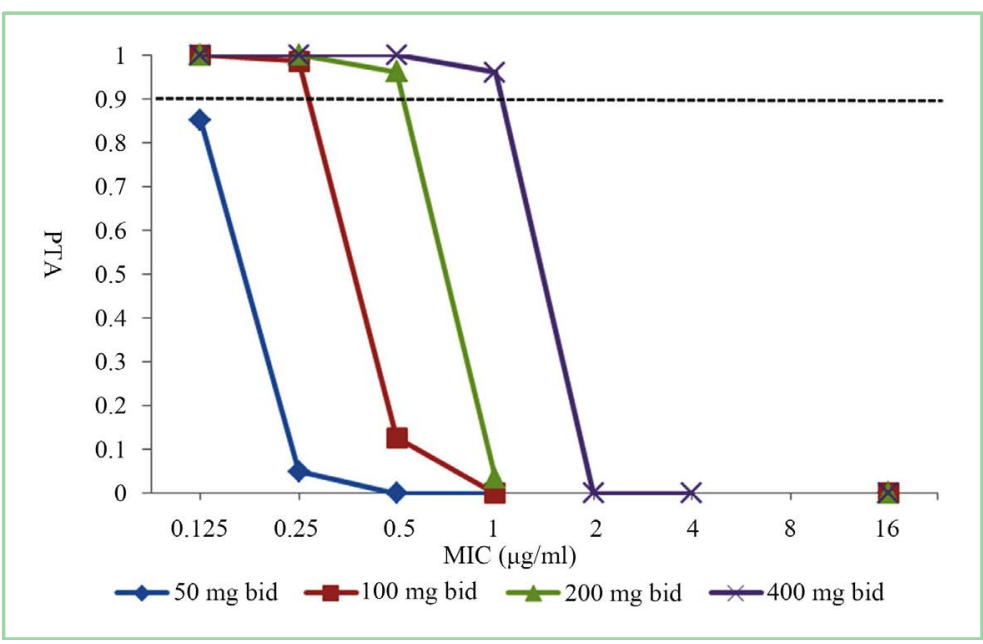

(b) 


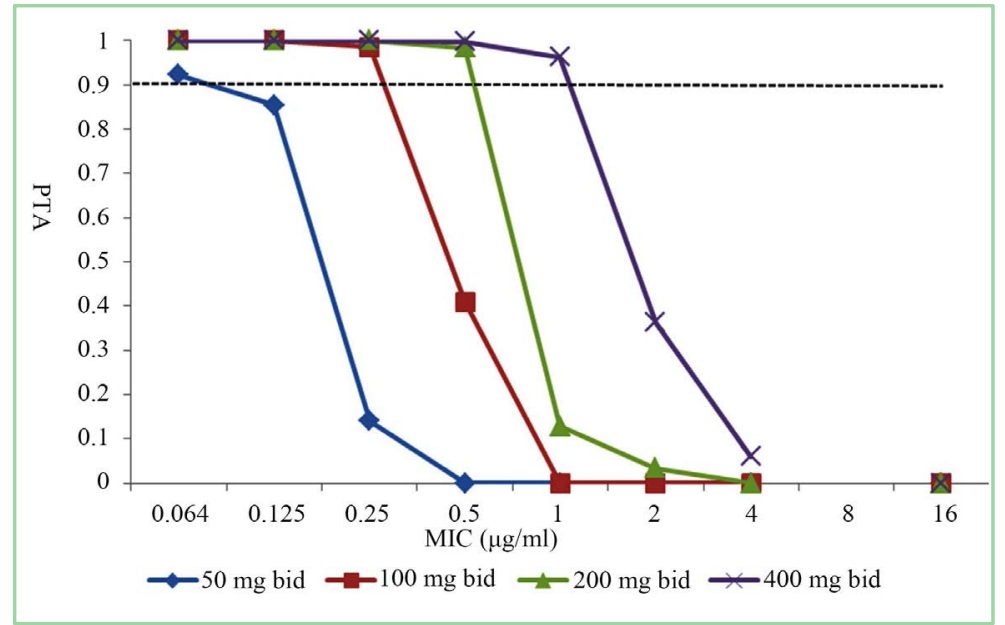

(c)

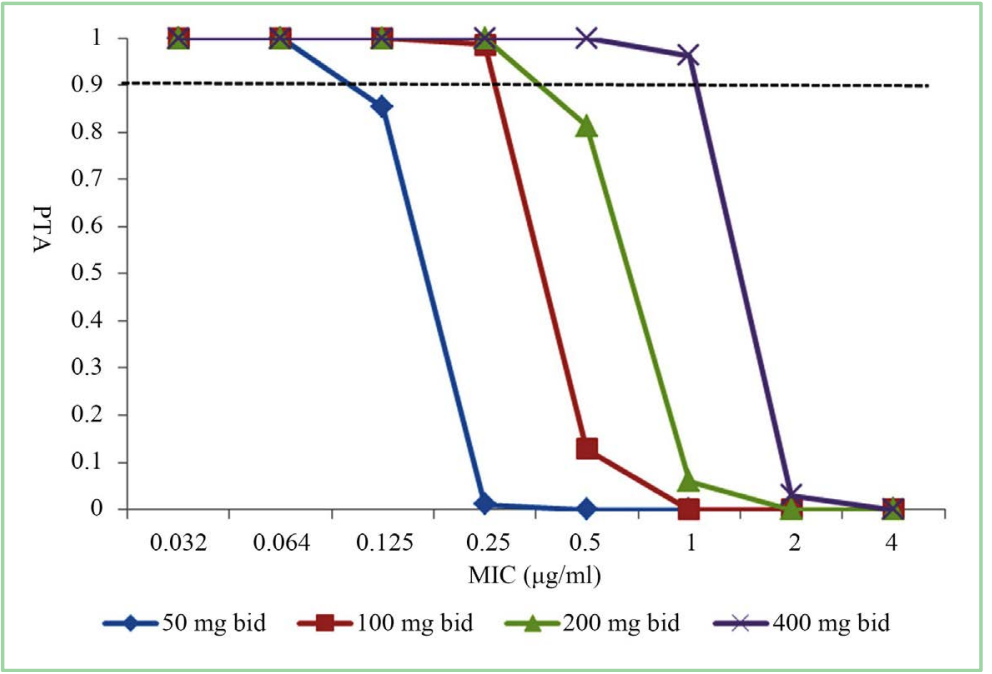

(d)

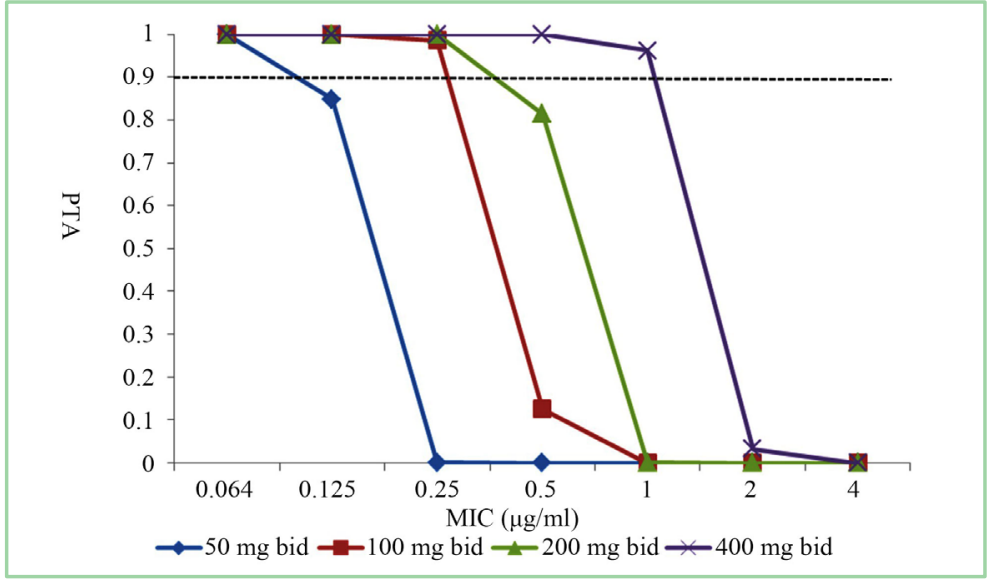

(e)

Figure 1. PTA of posaconazole estimated at different administration dosage in patients with mucormycosis. Different letters represent different Mucorales ((a), Lichtheimia corymbifera, (b), Rhizopus arrhizus, (c), Rhizopus microsporus, (d), Mucor sp., and (e), Rhizomucor pusillus). 


\section{Discussion}

Currently, the antifungal studies on mucormycosis were limited. Considering the range of fungal pathogens and the limited availability of antifungal agents, it was recommended to conduct in vitro antifungal susceptibility tests for individual isolates of invasive fungal infections in high-risk patients, to optimise treatment strategies and detect drug-resistant strains [11]. Posaconazole was recommended for treating the patients with mucormycosis by the European Society for Clinical Microbiology and the European Confederation of Medical Mycology [14].

Posaconazole was a concentration-dependent antifungal drug, which the main parameter of PK/PD analysis was the AUC/MIC. According to before the neutropenia model of mouse, AUC/MIC was set more than 100 for as to the target values of posaconazole for treatment mucormycosis [15]. In this study, the results of Monte Carlo simulation of multiple administration schemes of posaconazole showed that the optimal dosage administration for five Mucorales were different recommended dosage. The Monte Carlo simulation of posaconazole showed that the best dosage regimen for Lichtheimia corymbifera was $200 \mathrm{mg}$ orally q12h. The optimal dosage of posaconazole for Rhizopus microsporus and Rhizomucor pusillus was $400 \mathrm{mg}$ orally $\mathrm{q} 12 \mathrm{~h}$. However, the dosage of posaconazole including from 50 to $400 \mathrm{mg}$ orally $\mathrm{q} 12 \mathrm{~h}$ had poor antifungal effects on Rhizopus arrhizus and Mucor sp. in this Monte Carlo simulation. Lewis RE et al. reported that the recommended maximum dose of posaconazole was $400 \mathrm{mg}$ orally q12h because of the lack of clinical dosage of it [9]. When the fungus culture results were shown to be the fungus mentioned above, the clinical decision would be changed to use other antifungal drugs. Therefore, clinicians needed to conduct fungus culture and measure MIC in the treatment of mucormycosis, to rationally use posaconazole dose, avoid the occurrence of its resistance caused by practical use, reduce adverse reactions and the cost of a drug, and improve the chances of curing patients. Moreover, early TDM was necessary for dosing adjustment with posaconazole because of the posaconazole with insufficient PTA at the beginning of treatment [15].

Although the Monte Carlo simulation based on certain PK and strain data was convenient to optimise the type and dose of antibacterial drugs for treatment, the results of this study also had some limitations. First of all, because there were many kinds of pathogenic bacteria about mucormycosis [16]. The sample size was limited, the only reaction of certain people. Secondly, the Monte Carlo simulation in this paper only considered the serum concentration of posaconazole, while in clinical practice, the MIC value obtained from non-blood samples such as sputum or effusion was not included, which results in the fact that the MIC value of samples for some site infections was inconsistent. Finally, the source of bacterial MIC value is only the data of some regions and previous years, so the sample determination results of this part were not appropriate and cannot reflect the change of future trend. 


\section{Conclusion}

In summary, Monte Carlo simulation was simple, with optimisation design, economy, safety and predictability of clinical characteristics in determining antimicrobial activity. In this study, none of the simulated posaconazole dosage regimens were effective against Rhizopus arrhizus and Mucor sp. For Rhizopus microsporus and Rhizomucor pusillus, high doses of posaconazole (400 mg orally q12h.) should be recommended and used for patients with mucormycosis. However, when patients with mucormycosis caused by Lichtheimia corymbifera, the dosage of posaconazole with $200 \mathrm{mg}$ orally q12h could achieve better target attainment. So patients with mucormycosis by using posaconazole were not only Monte Carlo simulation but also in combination with the clinical condition.

\section{Funding}

This study was funded by the Study and Development Fund for Sciences and Technology in Chengde City (No. 201701A086 and No. 201701A087).

\section{Data Availability Statement}

All data generated or analyzed during this study are included in this published article.

\section{Conflicts of Interest}

The authors declare no conflicts of interest regarding the publication of this paper.

\section{References}

[1] Dannaoui, E. (2017) Antifungal Resistance in Mucorales. International Journal of Antimicrobial Agents, 50, 617-621.

https://doi.org/10.1016/j.ijantimicag.2017.08.010

[2] Farmakiotis, D. and Kontoyiannis, D.P. (2016) Mucormycoses. Infectious Disease Clinics of North America, 254, 143-163. https://doi.org/10.1016/j.idc.2015.10.011

[3] Skiada, A., Pagano, L., Groll, A., Zimmerli, S., Dupont, B., Lagrou, K., Lass-Florl, C., Bouza, E., Klimko, N., Gaustad, P., Richardson, M., Hamal, P., Akova, M., Meis, J.F., Rodriguez-Tudela, J.L., Roilides, E., Mitrousia-Ziouva, A. and Petrikkos, G. (2011) European Confederation of Medical Mycology Working Group on Zygomycosis. Zygomycosis in Europe: Analysis of 230 Cases Accrued by the Registry of the European Confederation of Medical Mycology (ECMM) Working Group on Zygomycosis between 2005 and 2007. Clinical Microbiology and Infection, 17, 1859-1867. https://doi.org/10.1111/j.1469-0691.2010.03456.x

[4] Tribble, D.R. and Rodriguez, C.J. (2014) Combat-Related Invasive Fungal Wound Infections. Current Fungal Infection Reports, 8, 277-286. https://doi.org/10.1007/s12281-014-0205-y

[5] Lee, S.C., Billmyre, R.B., Li, A., Carson, S., Sykes, S.M., Huh, E.Y., Mieczkowski, P., Ko, D.C., Cuomo, C.A. and Heitman, J. (2013) Analysis of a Foodborne Fungal Pathogen Outbreak: Virulence and Genome of a Mucor circinelloides Isolates from Yogurt. mBio, 5, e01390-14. https://doi.org/10.1128/mBio.01390-14

[6] Walther, G., Pawłowska, J., Alastruey-Izquierdo, A., Wrzosek, M., Rodriguez-Tudela, J.L., Dolatabadi, S., Chakrabarti, A. and de Hoog, G.S. (2013) DNA Barcoding in 
Mucorales: An Inventory of Biodiversity. Persoonia, 30, 11-47. https://doi.org/10.3767/003158513X665070

[7] Vehreschild, J.J., Birtel, A., Vehreschild, M.J., Liss, B., Farowski, F., Kochanek, M., Sieniawski, M., Steinbach, A., Wahlers, K., Fätkenheuer, G. and Cornely, O.A. (2013) Mucormycosis Treated with Posaconazole: Review of 96 Case Reports. Critical Reviews in Microbiology, 39, 310-324. https://doi.org/10.3109/1040841X.2012.711741

[8] Xiao, L., Madison, V., Chau, A.S,, Loebenberg, D., Palermo, R.E. and McNicholas, P.M. (2014) Three-Dimensional Models of Wild-Type and Mutated Forms of Cytochrome p450 14 Alpha-Sterol Demethylases from Aspergillus fumigatus and Candida albicans Provide Insights into Posaconazole Binding. Antimicrobial Agents and Chemotherapy, 48, 568-574. https://doi.org/10.1128/AAC.48.2.568-574.2004

[9] Lewis, R.E., Albert, N.D. and Kontoyiannis, D.P. (2014) Comparative Pharmacodynamics of Posaconazole in Neutropenic Murine Models of Invasive Pulmonary Aspergillosis and Mucormycosis. Antimicrobial Agents and Chemotherapy, 58, 6767-6772. https://doi.org/10.1128/AAC.03569-14

[10] Courtney, R., Pai, S., Laughlin, M., Lim, J. and Batra, V. (2003) Pharmacokinetics, Safety, and Tolerability of Oral Posaconazole Administered in Single and Multiple Doses in Healthy Adults. Antimicrobial Agents and Chemotherapy, 47, 2788-2795. https://doi.org/10.1128/AAC.47.9.2788-2795.2003

[11] Borman, A.M., Fraser, M., Palmer, M., Szekely, A., Houldsworth, M., Patterson, Z. and Johnson, E.M. (2017) MIC Distributions and Evaluation of Fungicidal Activity for Amphotericin B, Itraconazole, Voriconazole, Posaconazole and Caspofungin and 20 Species of Pathogenic Filamentous Fungi Determined Using the CLSI Broth Microdilution Method. Journal of Fungi (Basel, Switzerland), 31, pii: E27. https://doi.org/10.3390/jof3020027

[12] Wang, Y., Zhao, J.Y., Yao, Y.H., Hu, J.H., Hu, J.L., Xiao, X. and Zhao, Y.W. (2020) Optimization of Dalbavancin in Patients with Hepatic or Renal Impairment. Pharmacology \& Pharmacy, 11, 29-37. https://doi.org/10.4236/pp.2020.112004

[13] Dolton, M.J., Brüggemann, R.J., Burger, D.M. and McLachlan, A.J. (2014) Variability in Posaconazole Exposure Using an Integrated Population Pharmacokinetic Analysis. Antimicrob Agents Chemother, 58, 6879-6885. https://doi.org/10.1128/AAC.03777-14

[14] Cornely, O.A., Arikan-Akdagli, S., Dannaoui, E., Groll, A.H., Lagrou, K., Chakrabarti, A., Lanternier, F., Pagano, L., Skiada, A., Akova, M., Arendrup, M.C., Boekhout, T., Chowdhary, A., Cuenca-Estrella, M., Freiberger, T., Guinea, J., Guarro, J., de Hoog, S., Hope, W., Johnson, E., Kathuria, S., Lackner, M., Lass-Flörl, C., Lortholary, O., Meis, J.F., Meletiadis, J., Muñoz, P., Richardson, M., Roilides, E., Tortorano, A.M., Ullmann, A.J., van Diepeningen, A., Verweij, P. and Petrikkos, G. (2014) ESCMID and ECMM Joint Clinical Guidelines for the Diagnosis and Management of Mucormycosis 2013. Clinical Microbiology and Infection, 3, 5-26.

https://doi.org/10.1111/1469-0691.12371

[15] Leelawattanachai, P., Montakantikul, P., Nosoongnoen, W. and Chayakulkeeree, M. (2019) Pharmacokinetic/Pharmacodynamic Study of Posaconazole Delayed-Release Tablet in a Patient with Coexisting Invasive Aspergillosis and Mucormycosis. The rapeutics and Clinical Risk Management, 15, 589-595.

https://doi.org/10.2147/TCRM.S203625

[16] Chowdhary, A., Singh, P.K., Kathuria, S., Hagen, F. and Meis, J.F. (2015) Comparison of the EUCAST and CLSI Broth Microdilution Methods for Testing Isavuconazole, Posaconazole, and Amphotericin B against Molecularly Identified Mucorales Species. Antimicrobial Agents and Chemotherapy, 59, 7882-7887.

https://doi.org/10.1128/AAC.02107-15 